\title{
Toxicity profile and clinical outcomes in locally advanced head and neck cancer patients treated with induction chemotherapy prior to concurrent chemoradiation
}

\author{
ERIC C. KO ${ }^{1}$, ERIC M. GENDEN ${ }^{2}$, KRZYSZTOF MISIUKIEWICZ ${ }^{3}$, PETER M. SOM ${ }^{4}$, \\ LALE KOSTAKOGLU $^{4}$, CHIEN-TING CHEN ${ }^{1}$, STUART PACKER ${ }^{3}$ and JOHNNY KAO ${ }^{1,5}$ \\ Departments of ${ }^{1}$ Radiation Oncology, ${ }^{2}$ Otolaryngology, ${ }^{3}$ Medical Oncology and \\ ${ }^{4}$ Radiology, Mount Sinai Medical Center, New York, NY, USA
}

Received August 10, 2011; Accepted September 12, 2011

DOI: $10.3892 / o r .2011 .1512$

\begin{abstract}
The use of induction chemotherapy prior to chemoradiation for locally advanced head and neck squamous cell carcinoma (LA-HNSCC) remains controversial. We explored whether toxicity from induction chemotherapy influenced the delivery of concurrent chemoradiation. Among 171 consecutive previously unirradiated patients with HNSCC treated with combined chemotherapy and radiation, we identified 66 patients with stage III-IVB head and neck carcinoma who were treated with induction chemotherapy prior to planned chemoradiation. The most common induction regimen was docetaxel, cisplatin and 5-FU (TPF; 80\%) for 2 to 3 cycles. Mean radiation dose was 72 Gy (range, 36-75 Gy). Concurrent chemotherapy regimens included cisplatin (26\%), cetuximab (5\%) and 5-fluorouracil/hydroxyurea (65\%)-based regimens. At a median follow-up of 27 months (range, 9-56 months), the 2-year locoregional control and distant control rates were 85 and $86 \%$, respectively. The 2-year disease-free survival and overall survival rates were 74 and $80 \%$, respectively. Although there were no grade 5 toxicities during induction chemotherapy, $26 \%$ of patients required hospitalization for adverse events, including 5\% needing intensive care. The most common high grade adverse events were grade 4 neutropenia (21\%) and neutropenic fever (17\%). Six percent of patients were unable to tolerate concurrent chemotherapy. The 2-year disease-free survival was significantly higher in patients able to complete induction and concurrent chemoradiation as planned (83 vs. $27 \%, \mathrm{p}<0.001)$. Induction chemotherapy followed by concurrent chemoradiation results in promising survival rates in our cohort of advanced head and neck carcinoma patients. Due to
\end{abstract}

Correspondence to: Dr Johnny Kao, ${ }^{5}$ Present address: Florida Radiation Oncology Group, 717 West Robertson Street, Brandon, FL 33511, USA

E-mail: johnnykaomd@gmail.com

Key words: induction chemotherapy, head and neck cancer, concurrent chemoradiation, toxicity, adverse events severe toxicities in a subset of patients, this strategy is only recommended in selected high-risk patients who are carefully followed by an experienced multidisciplinary team.

\section{Introduction}

In the era of more effective concurrent chemoradiation regimens for locally advanced head and neck cancer, there has been a reversal of the patterns of failure, with distant metastases occurring more frequently than locoregional relapse $(1,2)$. Approximately $15-30 \%$ of patients with locally advanced head and neck cancer ultimately develop distant failure despite concurrent chemoradiation (3). Patients considered at greatest risk for distant relapse are those with advanced N2b-N3 nodal disease or unresectable disease (4). The ability of induction chemotherapy to prevent distant metastases and improve overall survival remains controversial, although some data from retrospective and prospective studies support a beneficial effect $(2,5-7)$. In contrast, most randomized trials fail to demonstrate a significant effect of concurrent chemotherapy on distant metastases, although a modest reduction cannot be entirely ruled out (6,8-10). The emergence of taxane-based induction chemotherapy regimens, which demonstrated improved efficacy compared to older regimens, has strengthened the rationale for sequential chemotherapy followed by chemoradiation $(7,11,12)$.

Despite the considerable promise of induction chemotherapy, there remain significant concerns that have limited its widespread adoption. First, severe toxicity occurring during induction chemotherapy may delay or prevent the administration of definitive radiotherapy $(3,13)$. In addition, as concurrent chemoradiation is already associated with high rates of acute and late toxicity, there are concerns that the toxicity from induction chemotherapy may limit the ability of patients to tolerate the toxicity of concurrent chemoradiation $(1,9,14)$. In the TAX 324 study, $27 \%$ of patients were unable to complete the treatment regimen, including $8 \%$ of patients who ultimately did not receive radiation.

We undertook the current study to better define the toxicities that may potentially limit the use of induction chemotherapy followed by concurrent chemoradiation for the 
Table I. Characteristics of the advanced head and neck carcinoma patients enrolled in study.

\begin{tabular}{|c|c|}
\hline & $\mathrm{N}(\%)$ \\
\hline \multicolumn{2}{|l|}{ Age } \\
\hline Median & 60 \\
\hline Range & $18-82$ \\
\hline \multicolumn{2}{|l|}{ Gender } \\
\hline Male & $53(80)$ \\
\hline Female & $13(20)$ \\
\hline \multicolumn{2}{|l|}{ Performance status } \\
\hline 0 & $24(36)$ \\
\hline 1 & $35(53)$ \\
\hline $2-3$ & $7(11)$ \\
\hline \multicolumn{2}{|l|}{ Race } \\
\hline White & $46(70)$ \\
\hline Black & $6(9)$ \\
\hline Hispanic & $11(17)$ \\
\hline Asian & $3(5)$ \\
\hline \multicolumn{2}{|l|}{ Smoking } \\
\hline None & $16(24)$ \\
\hline $\begin{array}{l}\leq 10 \text { pack years (including pipe, } \\
\text { cigar, betel nut, marijuana) }\end{array}$ & $12(18)$ \\
\hline 10.1-40 pack years & $15(14)$ \\
\hline$>40$ pack years & $19(29)$ \\
\hline Missing & $4(6)$ \\
\hline \multicolumn{2}{|l|}{ Primary site } \\
\hline Sinonasal & $2(3)$ \\
\hline Nasopharynx & $7(13)$ \\
\hline Oropharynx & $43(65)$ \\
\hline Oral cavity & $1(2)$ \\
\hline Salivary gland & $1(2)$ \\
\hline Larynx & $4(6)$ \\
\hline Hypopharynx & $7(11)$ \\
\hline Unknown primary & $1(2)$ \\
\hline \multicolumn{2}{|l|}{ AJCC stage } \\
\hline III & $4(6)$ \\
\hline IVA & 49 (74) \\
\hline IVB & $13(20)$ \\
\hline
\end{tabular}

definitive treatment of advanced head and neck carcinomas. In addition, we report our initial clinical outcomes, including locoregional and distant control as well as disease-free and overall survival. Lastly, by analysis of patient variables, we sought to identify useful prognostic factors that define subsets of patients who benefit from this treatment approach.

\section{Materials and methods}

Patients. Between August 2005 and June 2009, 171 consecutive patients with histologically confirmed stage III-IVB squamous
Table II. T and N staging of the patients.

\begin{tabular}{lrrrrrr}
\hline & Tx & T1 & T2 & T3 & T4 & Total \\
\hline N0 & & & & 1 & 4 & 5 \\
N1 & & & 2 & 1 & 7 & 10 \\
N2a & & 2 & 4 & 1 & & 7 \\
N2b & 1 & 2 & 9 & 4 & 7 & 23 \\
N2c & & 2 & 1 & 5 & 4 & 12 \\
N2 (npx only) & & & 1 & & & 1 \\
N3 & & 2 & 3 & 1 & 2 & 8 \\
Total & 1 & 8 & 20 & 13 & 24 & 66 \\
\hline
\end{tabular}

cell carcinoma of the head and neck were treated at Mount Sinai Medical Center (New York, NY) with chemotherapy and radiation (Table I). A cohort of 66 patients (39\%) were treated with induction chemotherapy followed by definitive local therapy. In general, patients were selected for induction chemotherapy on the basis of their increased risk of distant metastases, with $94 \%$ of patients having T4 and/or N2-3 disease (Table II). This cohort includes 3 patients with synchronous non-metastatic lung, esophagus and bladder primary tumors. Prior to initiation of treatment, all patients underwent multidisciplinary evaluation, including complete history, physical examination, fiberoptic nasolaryngoscopy, laboratory evaluation (including complete blood count with differential, blood chemistries, and liver function tests), imaging (CT neck, and/ or PET or CT chest) and dental evaluation. The institutional review board approved this retrospective review.

Induction chemotherapy. All patients received platinum-based induction chemotherapy. Most patients (80\%) were planned to receive docetaxel $\left(75 \mathrm{mg} / \mathrm{m}^{2}\right.$ on day 1$)$, cisplatin $\left(75 \mathrm{mg} / \mathrm{m}^{2}\right.$ on day 1) and 5 -FU (750 mg/m $\mathrm{m}^{2}$ on days 1-5) for two to three 21 day cycles $(12,13)$. Carboplatin and taxol $(17 \%)$, cisplatin and 5-fluorouracil (2\%), and single agent cisplatin (2\%) were also used. All patients who completed induction chemotherapy underwent restaging with $\mathrm{CT}$ of the neck.

IMRT technique. Radiation treatment was delivered with intensity modulated radiation therapy (IMRT). An extended-field IMRT technique was used to cover target volumes. High-risk planning treatment volume (PTV) consisted of the pre-chemotherapy gross tumor volume (GTV) or sites of microscopic positive margin $+1.2 \mathrm{~cm}$ margin. The intermediate-risk PTV consisted of the tumor bed and first echelon of clinically uninvolved lymph nodes. The lower-risk PTV included the uninvolved ipsilateral supraclavicular fossa, contralateral neck and bilateral retropharyngeal nodes. Standard normal tissue constraints were utilized.

Patients underwent $\mathrm{CT}$ simulation in the radiation oncology suite with PET fusion, when available. Patients were treated supine and rigidly immobilized with a custom Aquaplast mask and customized shoulder immobilization. Patients were planned using Eclipse version 8.0 and treated on a Varian 21EX linear accelerator with $6 \mathrm{MV}$ photons, using dynamic multileaf collimation. 


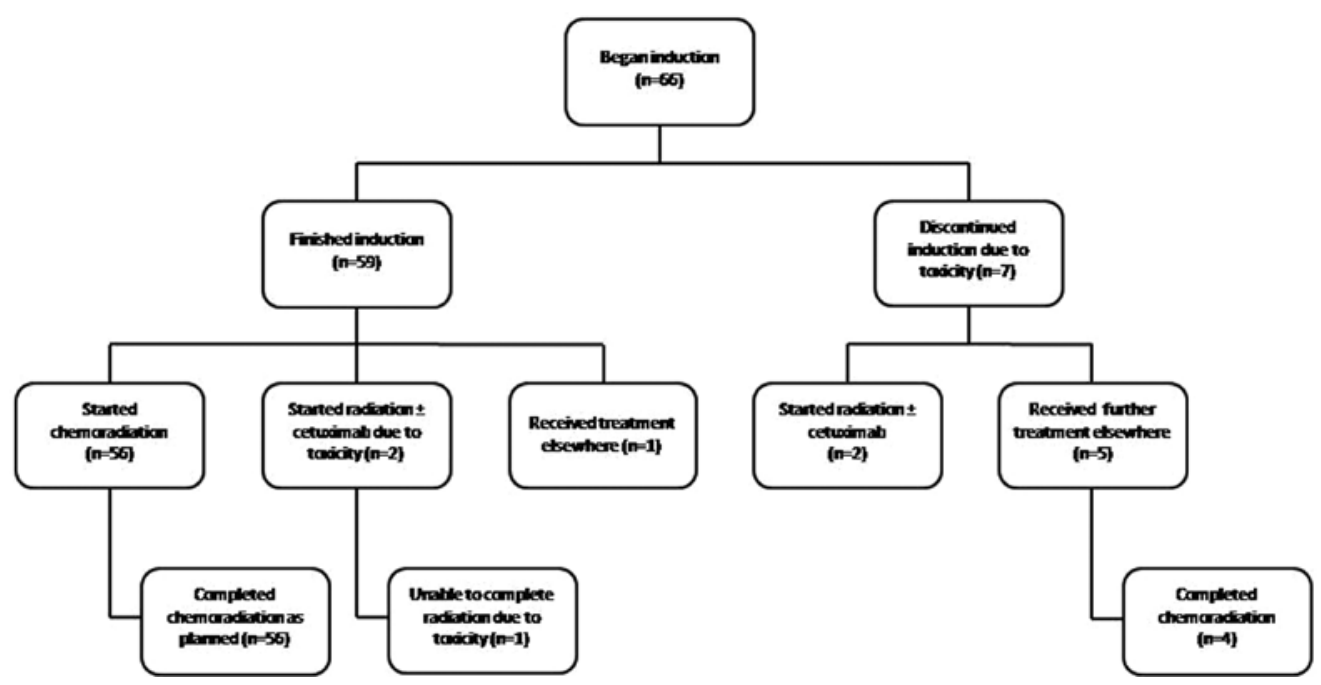

Figure 1. Numbers of patients who completed induction chemotherapy and concurrent chemoradiation per protocol.

Concurrent chemoradiation. Concurrent chemotherapy consisted of 5-fluorouracil (continuous infusion $600 \mathrm{mg} / \mathrm{m}^{2}$ on days 1-5), hydroxyurea (500 mg BID on days 1-5), and either cetuximab $\left(250 \mathrm{mg} / \mathrm{m}^{2}\right.$ on day 1$)$ or paclitaxel (dose $100 \mathrm{mg} / \mathrm{m}^{2}$ on day 1) on weeks $1,3,5,7$, and 9 in $65 \%$ of treated patients $(15,16)$. Weekly cisplatin $\left(40 \mathrm{mg} / \mathrm{m}^{2}\right)$, cetuximab $\left(400 \mathrm{mg} / \mathrm{m}^{2}\right.$ loading dose, followed by $250 \mathrm{mg} / \mathrm{m}^{2}$ ) or no concurrent chemotherapy was used in 26,5 , and $5 \%$ of patients, respectively.

Surgery. Limited surgery was performed in 9 patients (14\%) prior to chemoradiation. This included simple tonsillectomy with or without selective neck dissection $(n=5)$, biopsy of the primary site with neck dissection $(n=3)$, and endoscopic sinus resection $(n=1)$. One patient with ethmoid sinus carcinoma with extensive intracranial extension underwent craniofacial resection after induction chemotherapy.

Toxicity assessment. The primary endpoint of this study was tolerability and compliance with planned induction chemotherapy and concurrent chemoradiation. All toxic events were scored using the NCI CTCAE v. 3.0. Patients were prospectively followed by weekly head and neck cancer management rounds. Patient dropout and hospital and intensive care unit admissions were scored separately.

Clinical follow-up and statistical methods. All results were reported using intent to treat principles. Of the 60 patients treated with radiation in our institution, all underwent multidisciplinary follow-up every 2-3 months. This was often supplemented with PET/CT monitoring. For the 6 patients who left our institution after induction chemotherapy, follow-up was obtained via telephone supplemented with electronic medical record and social security death index searches.

Survival and time to progression were estimated with Kaplan-Meier plots. Clinical outcomes were stratified by prognostic factors and significance was assessed with the logrank test. A Cox proportional hazards model of disease-free survival, taking into account age, gender, race, performance status, tumor stage, nodal stage, smoking history, primary site, and ability to complete the planned treatment, was utilized for multivariate analysis.

\section{Results}

Adverse events and compliance during induction chemotherapy. Overall, 59 of 66 patients (89\%) completed induction chemotherapy (Fig. 1). Although there were no grade 5 toxicities during induction chemotherapy, $26 \%$ of patients required hospitalization for adverse events, including 5\% needing intensive care. The most common high grade adverse events were grade 4 neutropenia (21\%) and neutropenic fever (17\%) (Table III). Due to acute toxicity, 7 of 66 patients (11\%) were unable to complete the originally planned cycles of induction chemotherapy. Among these patients, 5 elected to transfer their care to another institution prior to completing their induction chemotherapy (Fig. 1).

Tolerance and compliance during concurrent chemoradiation. Due to toxicity occurring during induction chemotherapy, 3 of 59 patients $(5 \%)$ who completed induction chemotherapy were unable to receive concurrent chemoradiation. One patient elected to receive additional treatment at another facility, while 2 patients instead received concurrent cetuximab and radiotherapy. Among patients treated with chemoradiation in our institution, compliance with radiation was excellent, with all 56 patients receiving the planned dose of radiation. An unplanned treatment break of $\geq 7$ days occurred in $6 \%$ of patients, due to toxicity (3\%) or non-compliance (3\%). Overall, patients received $99 \%$ of the originally planned radiation dose. During radiation, rates of grade 3 mucositis and grade 3 dermatitis were $47 \%$ and $15 \%$, respectively (Table III). Fiftyfive percent of patients required the use of artificial nutrition. Rates of grade $\geq 3$ neutropenia and infection during chemoradiation were 41 and $7 \%$, respectively. Median weight loss during radiation was 17 pounds (range 0-40 lbs). There were no grade 5 toxicities during chemoradiation, although $3 \%$ of patients required intensive care admission. Overall, 56 of 66 patients $(85 \%)$ who began induction chemotherapy completed concurrent chemoradiation as originally planned. 
Table III. Incidence of toxicities in patients.

A, Toxicity during induction $(\mathrm{n}=66)$

\begin{tabular}{lccc}
\hline Toxicity during induction $(\mathrm{n}=66)$ & Grade $3(\%)$ & Grade $4(\%)$ & Grade $\geq 3(\%)$ \\
\hline Anemia & $7(11)$ & $0(0)$ & $1(2)$ \\
Thrombocytopenia & $2(3)$ & $14(21)$ & $7(11)$ \\
Neutropenia & $13(20)$ & $2(3)$ & $27(41)$ \\
Metabolic abnormality & $17(26)$ & $1(2)$ & $19(29)$ \\
Elevated LFTs & $4(6)$ & $0(0)$ & $5(8)$ \\
Elevated amylase & $3(5)$ & $0(0)$ & $3(5)$ \\
Stomatitis & $2(3)$ & $0(0)$ & $2(3)$ \\
Nausea & $1(2)$ & $1(2)$ & $0(0)$ \\
Diarrhea & $2(3)$ & $1(2)$ \\
Non-neutropenic infection & $5(8)$ & $2(3)$ \\
Renal dysfunction & $1(2)$ & $0(0)$ & $3(5)$ \\
Altered mental status (stroke, dementia) & & $2(3)$ \\
\hline
\end{tabular}

B, Toxicity during chemoRT $(n=60)$

\begin{tabular}{lccc}
\hline Toxicity during chemoRT $(\mathrm{n}=60)$ & Grade $3(\%)$ & Grade $4(\%)$ & Grade $\geq 3(\%)$ \\
\hline Mucositis & $28(47)$ & $0(0)$ & $0(0)$ \\
Dermatitis & $9(15)$ & $2(3)$ & $9(15)$ \\
Infection (aspiration pneumonia, line infection) & $2(3)$ & $0(0)$ & $4(7)$ \\
Anemia & $13(22)$ & $0(0)$ & $7(12)$ \\
Neutropenia & $7(12)$ & $0(0)$ & $1(2)$ \\
Nausea & $1(2)$ & $0(0)$ & $1(2)$ \\
Failure to thrive & $1(2)$ & & \\
\hline
\end{tabular}
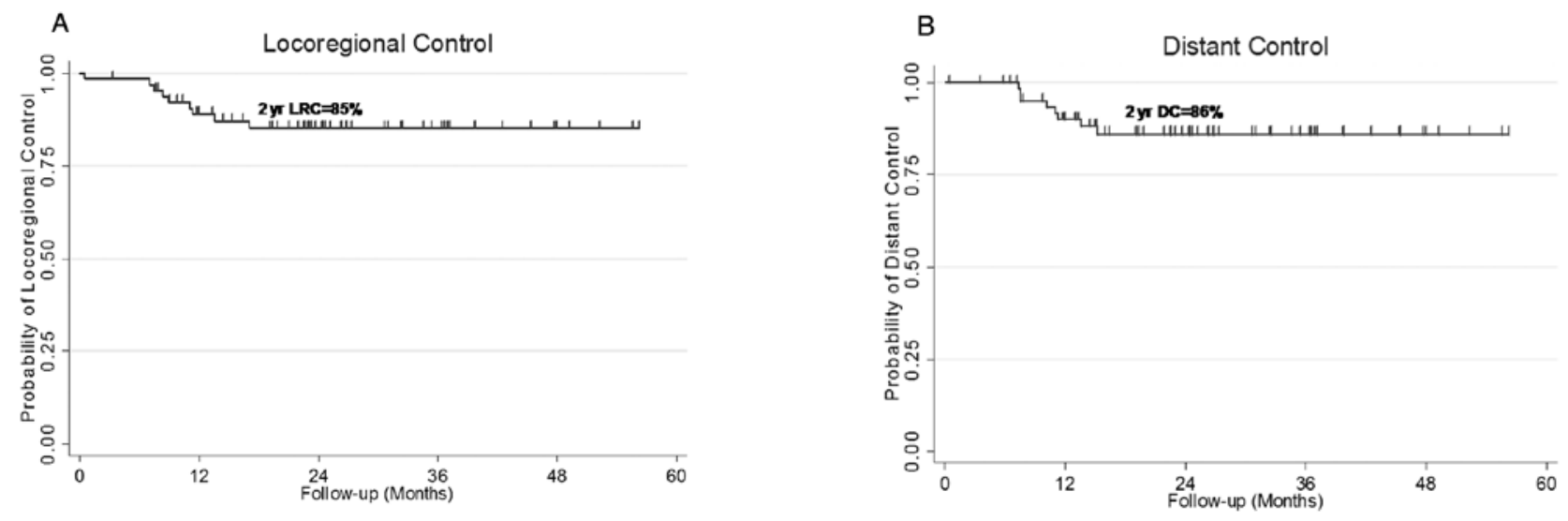

Figure 2. Locoregional and distant control. (A) Kaplan-Meier estimate of locoregional control among our cohort of 60 advanced head and neck carcinoma patients who underwent induction chemotherapy followed by concurrent chemoradiation. The 2-year locoregional control rate was $86 \%$. (B) Kaplan-Meier estimate of distant control of the same cohort of patients. The 2-year distant control rate was $85 \%$.

Clinical outcomes. The median follow-up in this cohort is 27 months (range, 9-56 months). A significant majority of these patients (94\%) have had >12 months of follow-up. By KaplanMeier analysis, the 2-year locoregional control and distant control rates were 86 and $85 \%$, respectively (Fig. 2). The 2-year disease-free survival and overall survival were 74 and $80 \%$, respectively (Fig. 3). Analysis of patterns of failure indicated local failure only (1\%), locoregional failure only (8\%), distant failure only (9\%), and locoregional and distant failure (5\%). Three percent of patients died from comorbid illness without disease failure, while $74 \%$ of patients are alive and without evidence of disease at the last follow-up.

Predictors of clinical outcome. When clinical outcomes were stratified by whether patients were able to complete all of their planned treatment, the 2-year locoregional 

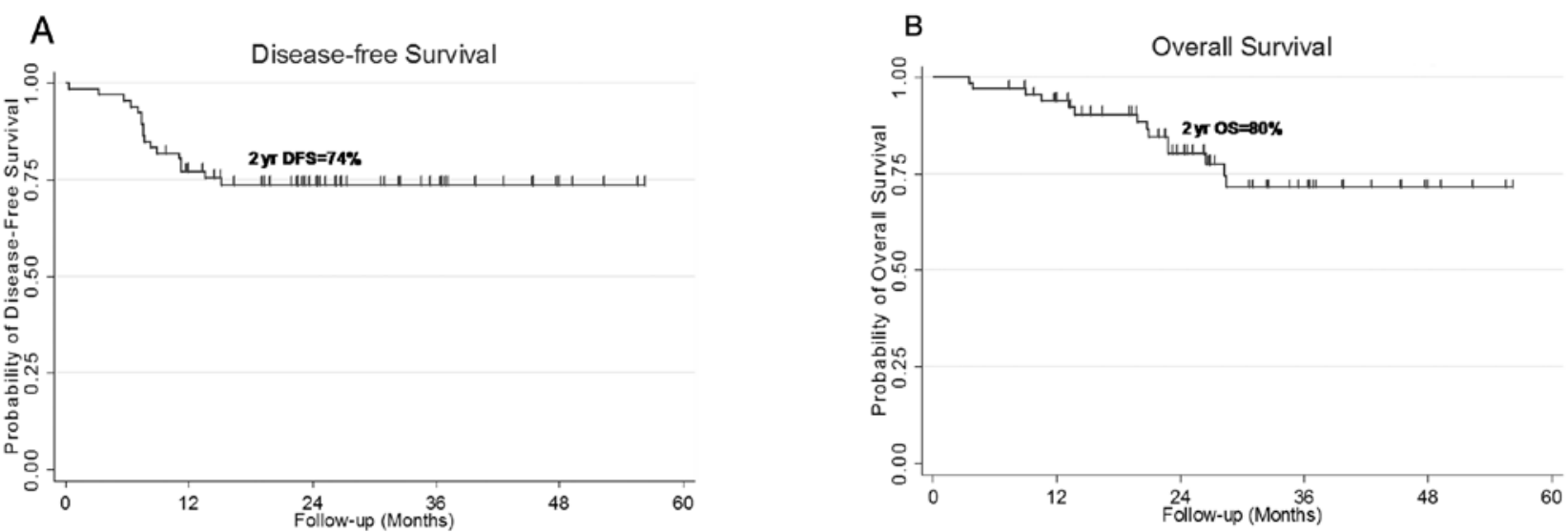

Figure 3. Disease-free and overall survival. (A) Kaplan-Meier estimate of disease-free survival among our cohort of 60 advanced head and neck carcinoma patients who underwent induction chemotherapy followed by concurrent chemoradiation. The 2-year disease-free survival was 74\%. (B) Kaplan-Meier estimate of overall survival of the same cohort of patients. The 2 -year overall survival was $80 \%$.
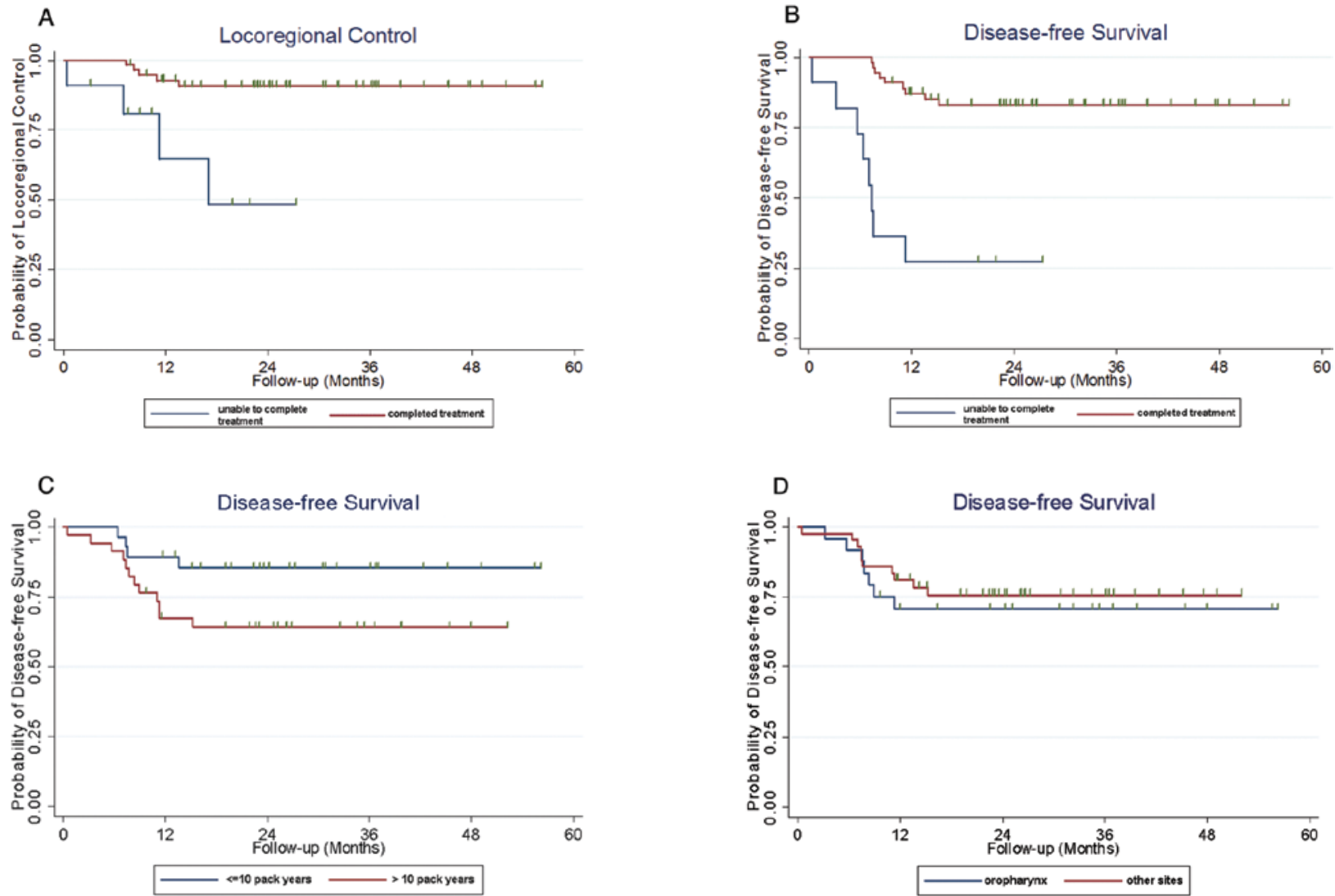

Figure 4. Prognostic factors predicting locoregional control and disease-free survival outcomes. Kaplan-Meier estimates of locoregional control or diseasefree survival among our cohort of advanced head and neck carcinoma patients were stratified by whether the patients successfully completed induction chemotherapy followed by concurrent chemoradiation (A and B), $>20$ vs. $\leq 20$ pack-years of smoking history (C), and oropharyngeal primary vs. other of head and neck primaries (D). A, the 2-year rate of locoregional control for patients who successfully completed treatment was 92 , vs. $48 \%$ for those who were unable to complete treatment $(\mathrm{p}<0.001)$. B, the 2 -year rate of disease-free survival for patients who successfully completed treatment was 84 , vs. $27 \%$ for those who were unable to complete treatment $(\mathrm{p}<0.001)$. C, the 2 -year rate of disease-free survival for patients with $\leq 20$ pack-year smoking history was 87 , vs. $60 \%$ for those with a $>20$ pack-year smoking history $(\mathrm{p}=0.02)$. D, the 2-year rate of disease-free survival for patients with an oropharyngeal primary was 75 , vs. $73 \%$ for those with other head and neck primaries $(\mathrm{p}=0.81)$.

control (91 vs. 48\%, p<0.001; Fig. 4A) and disease-free survival ( 83 vs. $27 \%$, p $<0.001$; Fig. 4B) were both noted to be significantly higher in those successfully completing treatment compared to those that did not. By log-rank analysis, patients with a minimal smoking history $(\leq 10$ pack years) had a trend towards improved disease-free survival than patients with a more extensive smoking history (85 vs. $64 \%$, p=0.06; Fig. 4C). Interestingly, having an oropharyngeal primary did not predict for improved disease-free survival in this cohort (76 vs. $71 \%, \mathrm{p}=0.6$; Fig. 4D). 
Table IV. Univariate and multivariate analyses of associations between patient factors and 2-year disease-free survival.

\begin{tabular}{|c|c|c|}
\hline Variable & $\mathrm{HR}(95 \% \mathrm{CI})$ & p-value \\
\hline \multicolumn{3}{|l|}{ Univariate analysis } \\
\hline Age & & 1.0 \\
\hline$<50$ (reference) & 1.0 & \\
\hline $50-69$ & 1.12 & \\
\hline$\geq 70$ & 1.27 & \\
\hline Race & & 0.66 \\
\hline White or other (reference) & 1.0 & \\
\hline Black & 0.7 & \\
\hline \multicolumn{3}{|l|}{ Gender } \\
\hline Male (reference) & 1.0 & 0.7 \\
\hline Female & 0.6 & \\
\hline Performance status & & 0.01 \\
\hline 0 (reference) & 1.0 & \\
\hline 1 & 1.7 & \\
\hline 2 & 3.6 & \\
\hline 3 & 10.2 & \\
\hline T stage & & 0.05 \\
\hline T0-2 (reference) & 1.0 & \\
\hline T3-4 & 2.8 & \\
\hline N stage & & 0.2 \\
\hline No & 0.0 & \\
\hline N1 & 1.8 & \\
\hline N2 (reference) & 1.0 & \\
\hline N3 & 2.4 & \\
\hline Primary tumor & & 0.6 \\
\hline Oropharynx (reference) & 1.0 & \\
\hline Non-oropharynx & 1.3 & \\
\hline Smoking history & & 0.06 \\
\hline$\leq 10$ pack years (reference) & 1.0 & \\
\hline$>10$ pack years & 2.8 & \\
\hline Treatment completion & & $<0.001$ \\
\hline Yes (reference) & 1.0 & \\
\hline $\begin{array}{l}\text { No (unable to receive concurrent } \\
\text { chemoRT or left institution) }\end{array}$ & 8.3 & \\
\hline \multicolumn{3}{|l|}{ Multivariate analysis } \\
\hline Age (continuous) & $1.02(0.97-1.08)$ & 0.5 \\
\hline Race (black race) & $0.94(0.11-8.11)$ & 1.0 \\
\hline Gender (female gender) & $1.51(0.29-7.82)$ & 0.6 \\
\hline Performance status (continuous) & $0.90(0.41-1.98)$ & 0.8 \\
\hline T stage (T3-4) & $2.78(0.71-10.92)$ & 0.2 \\
\hline $\mathrm{N}$ stage (N2-3) & $2.55(0.87-7.46)$ & 0.09 \\
\hline Primary site (oropharynx) & $0.56(0.18-1.76)$ & 0.3 \\
\hline Smoking history >10 pack years) & $2.36(0.58-9.66)$ & 0.2 \\
\hline \multicolumn{3}{|l|}{ Unable to complete treatment } \\
\hline Package & $10.5(2.52-43.48)$ & 0.001 \\
\hline
\end{tabular}

HR, hazard ratio; CI, confidence internal.
Univariate and multivariate analyses of factors associated with clinical outcome. In univariate and multivariate analyses, patients who were unable to complete the planned regimen of induction chemotherapy followed by concurrent chemoradiation fared significantly worse in their 2-year disease-free survival, compared to those who successfully completed this regimen (HR 10.5 in multivariate analysis; 95\% CI, 2.52-43.48; $\mathrm{p}=0.001$ ) (Table IV). By univariate analysis, performance status and $\mathrm{T}$ stage were independently and significantly associated with a difference in 2-year disease-free survival. Demographic factors such as age, race, and gender, and tumor factors such as $\mathrm{N}$ staging, and oropharyngeal vs. nonoropharyngeal primary, were not significantly associated with a difference in disease-free survival. The latter conclusion may be due, in part, to the limited number of patients in our study cohort. Aside from completion of treatment, which was significantly associated with improved 2-year disease-free survival, no other factors were significantly associated with an improved survival outcome. By multivariate analysis, patients with advanced T staging (HR 2.8; 95\% CI, 0.71-10.92; $\mathrm{p}=0.14$ ) or $\mathrm{N}$ staging (HR 2.6; 95\% CI, 0.87-7.46; $\mathrm{p}=0.2$ ) both appeared to trend towards an inferior 2-year disease-free survival. In addition, by multivariate analysis, patients with a smoking history of $>10$ pack-years, compared to $\leq 10$ pack-years, also trended towards an inferior 2-year disease-free survival (HR 2.4; $95 \%$ CI, 0.58-9.66; $\mathrm{p}=0.2$ ).

\section{Discussion}

Based on multiple randomized trials and a recent metaanalysis, the current standard of care for locally advanced head and neck cancer is concurrent chemoradiation $(6,7)$. However, recent studies of patterns of failure suggest that distant metastases are more common than locoregional recurrence in patients treated with effective chemoradiation regimens $(1,2)$. The use of taxane-based induction chemotherapy followed by concurrent chemotherapy and radiation has emerged as a rational approach to address the competing risks of locoregional and distant relapse $(11,13)$. To date, the TAX 324 regimen has represented the best studied approach to combining induction chemotherapy with concurrent chemoradiation $(12,13)$. Following induction chemotherapy, low dose weekly carboplatin was used concurrently with radiotherapy due to concerns of poor tolerance of high dose cisplatin (13). With this regimen, $21 \%$ of patients were unable to complete protocol therapy, including $9 \%$ of patients who were unable to receive radiation secondary to toxicity or progression (13). Since concurrent carboplatin is considered suboptimal radiosensitizing chemotherapy for head and neck cancer, it was notable that concurrent chemotherapy with either weekly cisplatin or 5-fluorouracil and hydroxyurea-based concurrent chemotherapy, which are considered more active regimens, proved to be feasible in our experience (17-19).

Our study indicates a relatively high rate of adverse events occurring during induction chemotherapy, which is not unexpected with an aggressive multiagent chemotherapy regimen $(12,13)$. Almost $30 \%$ of patients required hospitalization for complications related to induction chemotherapy, which were most commonly high grade neutropenia, neutropenic fever, and metabolic abnormalities. Two patients required ICU 
admission, one of whom was diagnosed with a stroke and the other of whom had prolonged pancytopenia. However, among the patients who continued on our induction regimen, most ultimately recovered from their acute toxicities and were able to tolerate concurrent chemotherapy. Of note, there were no grade 5 toxicities in our cohort. Significantly, among patients who received radiation, over $95 \%$ were able to receive the prescribed dose and less than $10 \%$ had unplanned treatment breaks of over 7 days, indicating that the concurrent chemoradiation component of this approach is generally well tolerated.

Our findings regarding the toxicities of aggressive induction chemotherapy parallel those of other investigators. The TAX 324 study reported $56-83 \%$ grade $3-4$ neutropenia and $21-27 \%$ grade 3-4 mucositis during induction chemotherapy, and $37-38 \%$ grade 3-4 mucositis during chemoradiotherapy (13). In this study, $29-65 \%$ of the patients had treatment delays during induction chemotherapy. This appeared to be due to a high number of hematologic adverse events, particularly in the cisplatin-5-fluorouracil (PF) induction arm. A recent SWOG study suggested that concurrent cisplatin $\left(100 \mathrm{mg} / \mathrm{m}^{2}\right)$ and accelerated fractionation radiation therapy is poorly tolerated after intensive induction chemotherapy (20). In addition, a phase III Madrid trial demonstrated that only $49 \%$ of patients received protocol-established concurrent cisplatin and radiation following induction cisplatin, 5-fluorouracil \pm docetaxel, suggesting significant toxicity with this regimen in some patients (21).

Overall, our 2-year rates of locoregional control, distant control and overall survival were promising, particularly for the $85 \%$ of patients who were able to complete induction chemotherapy followed by concurrent chemoradiation as originally planned. The outcomes were less satisfactory for the few remaining patients who were unable to tolerate the planned regimen due to toxicity. Although there is a theoretical risk that a delay in treatment may result in a reduction in overall treatment efficacy, as determined by inferior disease control and/or survival outcomes, our study was limited in its ability to detect if the outcomes for this subset of patients was in fact worse. This is an important issue to address in future clinical studies, as it directly addresses the major critique of an induction chemotherapy strategy.

There are several related areas of investigation that are worthy of note. First, there has been interest in exploring the use of concurrent cetuximab and radiotherapy following induction chemotherapy (22). This approach may be less toxic and thus better tolerated than one with concurrent chemoradiation; however, to our knowledge there has not been a clinical trial to address this issue. Second, recent evidence indicates that human papillomavirus (HPV)-associated head and neck cancers respond more favorably to multiple treatment modalities, including surgery, radiation, and concurrent chemoradiation $(23,24)$. Hence, it is reasonable to expect this subset of patients to respond more favorably to our treatment regimen as well. However, as the HPV status of tumors from the head and neck patients included in our series was not tested, we could not validate this hypothesis in our current analysis.

We eagerly await results from the ongoing DeCIDE, PARADIGM and Madrid phase III trials, which are designed to test the effect of induction chemotherapy on overall survival in the setting of concurrent chemoradiation (25). In the interim, induction chemotherapy followed by concurrent chemoradiation is worthy of consideration in head and neck cancer patients with clinical T4b and/or N2b-N3 disease, who are predicted to be at high risk for distant metastasis (25).

\section{References}

1. Adelstein DJ, Saxton JP, Lavertu P, et al: Maximizing local control and organ preservation in stage IV squamous cell head and neck cancer with hyperfractionated radiation and concurrent chemotherapy. J Clin Oncol 20: 1405-1410, 2002.

2. Brockstein B, Haraf DJ, Rademaker AW, et al: Patterns of failure, prognostic factors and survival in locoregionally advanced head and neck cancer treated with concomitant chemoradiotherapy: a 9-year, 337-patient, multi-institutional experience. Ann Oncol 15: 1179-1186, 2004.

3. Brizel DM and Vokes EE: Induction chemotherapy: to use or not to use? That is the question. Semin Radiat Oncol 19: 11-16, 2009.

4. Garden AS, Asper JA, Morrison WH, et al: Is concurrent chemoradiation the treatment of choice for all patients with stage III or IV head and neck carcinoma? Cancer 100: 1171-1178, 2004.

5. Paccagnella A, Orlando A, Marchiori C, et al: Phase III trial of initial chemotherapy in stage III or IV head and neck cancers: a study by the Gruppo di Studio sui Tumori della Testa e del Collo. J Natl Cancer Inst 86: 265-272, 1994.

6. Forastiere AA, Goepfert H, Maor M, et al: Concurrent chemotherapy and radiotherapy for organ preservation in advanced laryngeal cancer. N Engl J Med 349: 2091-2098, 2003.

7. Pignon JP, le Maitre A, Maillard E and Bourhis J: Meta-analysis of chemotherapy in head and neck cancer (MACH-NC): an update on 93 randomised trials and 17,346 patients. Radiother Oncol 92: 4-14, 2009.

8. Cooper JS, Pajak TF, Forastiere AA, et al: Postoperative concurrent radiotherapy and chemotherapy for high-risk squamous-cell carcinoma of the head and neck. N Engl J Med 350: 1937-1944, 2004.

9. Brizel DM, Albers ME, Fisher SR, et al: Hyperfractionated irradiation with or without concurrent chemotherapy for locally advanced head and neck cancer. N Engl J Med 338: 1798-1804, 1998.

10. Adelstein DJ, Lavertu P, Saxton JP, et al: Mature results of a phase III randomized trial comparing concurrent chemoradiotherapy with radiation therapy alone in patients with stage III and IV squamous cell carcinoma of the head and neck. Cancer 88: 876-883, 2000

11. Vokes EE, Stenson K, Rosen FR, et al: Weekly carboplatin and paclitaxel followed by concomitant paclitaxel, fluorouracil, and hydroxyurea chemoradiotherapy: curative and organ-preserving therapy for advanced head and neck cancer. J Clin Oncol 21: 320-326, 2003.

12. Vermorken JB, Mesia R, Rivera F, et al: Platinum-based chemotherapy plus cetuximab in head and neck cancer. N Engl J Med 359: 1116-1127, 2008.

13. Posner MR, Hershock DM, Blajman CR, et al: Cisplatin and fluorouracil alone or with docetaxel in head and neck cancer. $\mathrm{N}$ Engl J Med 357: 1705-1715, 2007.

14. Machtay M, Moughan J, Trotti A, et al: Factors associated with severe late toxicity after concurrent chemoradiation for locally advanced head and neck cancer: an RTOG analysis. J Clin Oncol 26: 3582-3589, 2008

15. Kao J, Genden EM, Gupta V, et al: Phase 2 trial of concurrent 5-fluorouracil, hydroxyurea, cetuximab, and hyperfractionated intensity-modulated radiation therapy for locally advanced head and neck cancer. Cancer 117: 318-326, 2011.

16. Haraf DJ, Rosen FR, Stenson K, et al: Induction chemotherapy followed by concomitant TFHX chemoradiotherapy with reduced dose radiation in advanced head and neck cancer. Clin Cancer Res 9: 5936-5943, 2003.

17. Garden AS, Harris J, Vokes EE, et al: Preliminary results of Radiation Therapy Oncology Group 97-03: a randomized phase ii trial of concurrent radiation and chemotherapy for advanced squamous cell carcinomas of the head and neck. J Clin Oncol 22: 2856-2864, 2004. 
18. Fountzilas G, Ciuleanu E, Dafni U, et al: Concomitant radiochemotherapy vs. radiotherapy alone in patients with head and neck cancer: a Hellenic Cooperative Oncology Group Phase III Study. Med Oncol 21: 95-107, 2004.

19. Traynor AM, Richards GM, Hartig GK, et al: Comprehensive IMRT plus weekly cisplatin for advanced head and neck cancer: the University of Wisconsin experience. Head Neck 32: 599-606, 2010.

20. Adelstein DJ, Moon J, Hanna E, et al: Docetaxel, cisplatin, and fluorouracil induction chemotherapy followed by accelerated fractionation/concomitant boost radiation and concurrent cisplatin in patients with advanced squamous cell head and neck cancer: a Southwest Oncology Group phase II trial (S0216). Head Neck 32: 221-228, 2010.

21. Hitt R, Lopez-Pousa A, Martinez-Trufero J, et al: Phase III study comparing cisplatin plus fluorouracil to paclitaxel, cisplatin, and fluorouracil induction chemotherapy followed by chemoradiotherapy in locally advanced head and neck cancer. J Clin Oncol 23: 8636-8645, 2005.
22. Buiret G, Combe C, Favrel V, et al: A retrospective, multicenter study of the tolerance of induction chemotherapy with docetaxel, cisplatin, and 5-fluorouracil followed by radiotherapy with concomitant cetuximab in 46 cases of squamous cell carcinoma of the head and neck. Int J Radiat Oncol Biol Phys 77: 430-437, 2010.

23. Vu HL, Sikora AG, Fu S and Kao J: HPV-induced oropharyngeal cancer, immune response and response to therapy. Cancer Lett 288: 149-155, 2010.

24. Fakhry C, Westra WH, Li S, et al: Improved survival of patients with human papillomavirus-positive head and neck squamous cell carcinoma in a prospective clinical trial. J Natl Cancer Inst 100: 261-269, 2008

25. Salama JK, Seiwert TY and Vokes EE: Chemoradiotherapy for locally advanced head and neck cancer. J Clin Oncol 25: 4118-4126, 2007. 